\title{
Prevalence of Helicobacter pylori in Patients With Dyspepsia
}

\author{
Ramin Niknam ${ }^{1}$; Mehrdad Seddigh ${ }^{2}$; Mohammad Reza Fattahi ${ }^{1}$; Amirreza Dehghanian ${ }^{3}$; \\ Laleh Mahmoudi ${ }^{2,4,}$ \\ ${ }^{1}$ Gastroenterohepatology Research Center, Shiraz University of Medical Sciences, Shiraz, IR Iran \\ ${ }_{2}^{2}$ Pharmaceutical Science Research Center (PSRC), School of Pharmacy, Shiraz University of Medical Sciences, Shiraz, IR Iran \\ 3 Department of Pathology, School of Medicine, Shiraz University of Medical Sciences, Shiraz, IR Iran \\ ${ }^{4}$ Department of Clinical Pharmacy, School of Pharmacy, Shiraz University of Medical Sciences, Shiraz, IR Iran \\ ${ }^{*}$ Corresponding author: Laleh Mahmoudi, Department of Clinical Pharmacy, School of Pharmacy, Shiraz University of Medical Sciences, Shiraz, IR Iran. Tel: +98-7112424128, Fax: +98- \\ 7112424126, E-mail:Mahmoudi_l@sums.ac.ir
}

Received: July 2, 2013; Revised: February 19, 2014; Accepted: March 9, 2014

\begin{abstract}
Background: The prevalence rate of gastric infections caused by Helicobacter pylori is different in between as well as within various countries, including Iran.

Objectives: This study was aimed to evaluate the prevalence of H.pylori in Iranian adult patients with dyspepsia.

Patients and Methods: A total of 548 patients with dyspepsia referred to Namazi Hospital, a referral center for gastrointestinal diseases in Shiraz, south of Iran, were evaluated in a cross-sectional study. The diagnosis of dyspepsia was based on clinical findings. Upper gastrointestinal endoscopy was performed for all the patients and two antral biopsies were taken from all the included ones. The patients were categorized based on the endoscopic findings, to four groups:1-normal, 2-abnormal nonulcerative, 3-ulcerative, and 4-combination of 2 and 3. Two positive test results (Gram staining, rapid urease test, and culture) was considered as a positive-definite H. pylori infection for each patient.

Results: From the 548 included patients (238 males and 310 females), H. pylori was detected in 170 (31.2\%). The mean age was $40.38 \pm 15.39$ years old. $H$. pylori infection was detected in $26.1 \%$ of male and $34.8 \%$ of female patients and its prevalence increased with age. Eighty three (48.8\%) patients were positive for $\mathrm{H}$. pylori infection, of which, $12.4 \%$ had normal and $36.4 \%$ had abnormal nonulcerative endoscopic findings; $17.1 \%$ of patients were ulcerative and $34.1 \%$ had ulcer with or without concurrent abnormality.

Conclusions: Findings from this study showed a lower prevalence of $H$. pylori infection than other studies and its prevalence increased with age. There was no association between sex and infection. The most common endoscopic abnormality in H.pylori-positive patients was ulcerative lesion.
\end{abstract}

Keywords:Helicobacter pylori; Dyspepsia; Prevalence; Endoscopy

\section{Background}

Helicobacter pylori is a Gram-negative bacteria with strong association with upper gastrointestinal diseases such as peptic ulcer diseases (gastric ulcer, duodenal ulcer) and malignancies (gastric cancer, lymphoma), reported worldwide and in Iran (1-3). In developing countries, $H$. pylori is typically acquired in early childhood and may persist for a lifetime if left untreated $(4,5)$. In adults, the $H$. pylori infection rate increases with age $(6,7)$. Oraloral and fecal-oral routes are the most common modes of $H$. pylori transmission in developing countries. Less common modes of transmission are zoonotic and iatrogenic (such as endoscopic or through dental procedures) transmissions $(8,9)$.

In several previous studies, there were differences in the prevalence rate of $H$. pylori infection between countries $(1,10-14)$. It can be due to diverse contributing factors including socioeconomic status, geographical or living conditions, and ethnicity or location of each population (1). Dyspepsia is a medical condition characterized by chronic or recurrent pain in the upper abdomen, which is considered as one of the most common causes of patients' referrals to gastroenterology centers (15).

The definition of dyspepsia has been elusive with variety of proposed descriptions (16). Dyspepsia is defined based on the recent Asian Consensus Report as a symptom or set of symptoms that is (are) considered to originate from the gastroduodenal region. In this consensus report, the dyspepsia symptoms are epigastric pain, epigastric burning, postprandial fullness, early satiation, and others, including bloating in the upper abdomen, nausea, vomiting and belching. Chronic dyspeptic symptoms can be continuous, intermittent, or recurrent. Most of the consensus members agreed that symptom duration of equal or more than three months might be enough for diagnosis of dyspepsia. Functional dyspepsia is defined as chronic dyspeptic symptoms in the absence of organic, systemic, or metabolic condition (s), which is (are) likely to explain the symptoms (16). In the western world, the reported prevalence of dyspepsia ranged 25 $50 \%(17,18)$. In the south-western United States, dyspep-

Copyright (C 2014,Ahvaz Jundishapur University of Medical Sciences; Published by Kowsar Corp. This is an open-access article distributed under the terms of the Creative Commons Attribution License, which permits unrestricted use, distribution, and reproduction in any medium, provided the original work is properly cited. 
sia and $H$. pylori-related gastrointestinal diagnoses were remarkably common, with an annual incidence of 13 per 1000 individuals (15).

Alazmi et al. (12) demonstrated that the overall prevalence of patients with $H$. pylori infection and dyspepsia was $49.7 \%$ in Kuwait. In a systematic review by Amini et al. (19), the prevalence of dyspepsia in Iran varied between $2.2 \%$ to $29.9 \%$. Understanding the latest prevalence of $H$. pylori infection in patients with dyspepsia and determining its associations with endoscopic findings and pathologic changes in gastric mucosa can be useful for healthrelated purposes in every region and country. Some reports from Asia and Iran have demonstrated that the prevalence rate of $H$. pylori infection has declined rapidly in these regions $(10,11)$.

\section{Objectives}

This study was conducted to determine the prevalence rate of $H$.pylori infection among a population of adult patients with dyspepsia in Shiraz, south of Iran, using Gram staining, rapid urease test, and culture. We also hoped to find the rate of $H$. pylori infection in different endoscopic findings.

\section{Patients and Methods}

\subsection{Study Population}

After obtaining the approval of the university ethical committee (91-01-36-4703) as well as written informed consent from the patients, all the patients with dyspepsia attending Namazi Hospital in Shiraz; as a major referral center for gastrointestinal diseases in south of Iran, were recruited into the study between May and November 2012. The exclusion criteria for all the patients were age less than 18 , history of $H$. pylori eradication, history of consumption of proton pump inhibitors (PPIs), H2-receptor antagonists, antacids or antibiotics within four weeks prior to the endoscopic evaluation, and history of gastric or esophageal surgery.

Diagnosis of dyspepsia was based on clinical findings. We defined dyspepsia if the patient had one or more of these symptoms with duration of three months or longer: postprandial fullness, early satiation, epigastric pain, epigastric burning, bloating in the upper abdomen, nausea, vomiting, and belching (16). Upper gastrointestinal endoscopy was performed for all the patients with dyspepsia as an objective assessment for the diagnosis of causes was also performed. We categorized our patients based on the endoscopic finding into four groups:

1- normal, 2- abnormal nonulcerative (any evidence of mucosal lesion without ulcer, e.g. erosion, erythema, nodularity, atrophy, white plaque, and petechiae), 3- ulcerative, and 4-combination of 2 and 3. Two antral biopsies were performed for all the included patients with normal and abnormal endoscopy results. Biopsy was also performed for any abnormal lesion. After the endoscopy, the biopsy samples were transferred to the lab under an appropriate condition for pathology evaluation. All the samples were evaluated by rapid urease test and Gram staining for the presence of $H$. pylori. If one of these test results was negative, the biopsy samples were gently cultured using standard methods (11). We defined $H$. pylori as positive if two of these test results (Gram staining, rapid urease test, and culture) were positive.

\subsection{Statistical Analysis}

All the data were expressed as mean \pm standard error. Chi square test was used and significance was defined as a P value $<0.05$. Data analysis was performed with SPSS software (version 19, SPSS Inc., Chicago, Illinois, USA).

\section{Results}

The endoscopic findings and the $H$. pylori test results of 631 patients with dyspepsia were evaluated retrospectively in this cross-sectional study. A total of 548 patients with a mean age of $40.38 \pm 15.39$ years old were enrolled in the study and 83 patients were excluded. Of the included patients, 238 (43.4\%) were male and 310 (56.6\%) were female. There was no association between sex and infection $(\mathrm{P}=0.57)$. Of the 548 patients tested, $170(31 \%)$ were positive and 378 (69\%) were negative for $H$. pylori infection. $H$. pylori infection was observed in $26.1 \%$ of male and $34.8 \%$ of female patients (Table 1) and its prevalence increased with age. The overall prevalence of $H$. pylori infection had ha sharp increase from the middle-aged patients (5.3\% in 30-34-year-old and 5.8\% in 35-44-year-old patients) to $19.8 \%$ in those aged 45 or older (Table 1). As the endoscopy results indicated, 83 (48.8\%) H. pylori-positive patients had normal or abnormal nonulcerative endoscopic findings (groups 1 and 2) and 87 (51.2\%) had ulcer with or without concurrent abnormality (groups 3 and 4).

Table 1. Prevalence and Patterns of Endoscopic Findings in $H$. pylori Infection

\begin{tabular}{|ccc|}
\hline Variable & Patients (\%) & H.pylori-Positive, No. (\%) \\
\hline Gender & & $62(26.1)$ \\
\hline Male & $238(43.4)$ & $108(34.8)$ \\
\hline Female & $310(56.6)$ & $170(31)$ \\
\hline Total & $548(100)$ & \\
\hline Age & & $29(5.3)$ \\
$<35$ & $211(38.5)$ & $32(5.8)$ \\
\hline $35-44$ & $145(26.4)$ & $109(19.8)$ \\
\hline$>45$ & $192(35.1)$ & \\
\hline
\end{tabular}

\section{Discussion}

H. pylori infection is a major cause of gastroduodenal diseases; however, there have been different reports 
Niknam Ret al.

about the prevalence rate of this infection between countries $(1,10,11)$. Several studies from Asia and Middle East including China, South Korea, Iran, and Kuwait have shown that the prevalence of $H$. pylori has declined in these areas in the recent years (10-13). In the present study, H. pylori positivity was $31 \%$ (Table 1 ), which was surprisingly lower than other local reports $(2,3,11)$. In previous reports from Shiraz it was shown that more than $85 \%$ of Iranian adults carried IgG against $H$. pylori (2). In addition, histological evidence of $H$. pylori infection was recorded in $89.2 \%$ of biopsies in another report from north of Iran (3). Recently, in a survey conducted by Farshad et al. (11) in Iran, the prevalence of $H$. pylori in a total of 522 patients who underwent endoscopic evaluation was $47.9 \%$.

Interestingly, the results of this study showed a lower prevalence of $H$. pylori in comparison with other reports from Asia and Middle East. Goh (14) reported that the overall prevalence rate of $H$. pylori in a Malaysian population with dyspepsia was $49.0 \%$. In this study, two antral biopsies were performed for diagnosis of $H$. pylori, using a rapid urease test. In Alazmi et al.(12) research in Kuwait, an overall prevalence rate of $49.7 \%$ was reported in 362 patients with dyspepsia. In another report from India, Poddar and Yachha (6) demonstrated that almost $80 \%$ of the population in India was infected with $H$. pylori. Ahmed et al. (20) also showed that the overall prevalence of $H$. pylori in India was $80 \%$. In this study, the diagnosis of $H$. pylori was established by polymerase chain reaction (PCR) amplification of the 16S rRNA gene of $H$. pylori. As expected, similar to several other studies (10-13) our research showed that the prevalence of $H$. pylori was declined.

According to the present study, the most common endoscopic abnormality in $H$. pylori-positive patients was ulcerative lesion, comprising groups 3 and 4 (51.2\%) (Table 2). H. pylori infection can be responsible for gastroduodenal ulcer. This association has been shown in previous studies $(14,21)$. Goh $(14)$ reported that $H$. pylori prevalence differed based on endoscopic findings. Similar results were reported in 1990 by Soll (21). Regarding the sex, this study showed that $H$. pylori positivity had predominance in females; however, no significant association between sex and infection rate was shown. A vast variety of results have been reported regarding the predominance of either males or females; but, it is generally concluded that there is no significant association between the infection rate of $H$. pylori and sex $(1,12,20)$. There was a significant association between the $H$. pylori infection prevalence and age in our study, which was similar to some other researches $(1,12,20)$. The findings of this cross-sectional study on dyspepsia showed a lower prevalence of $H$. pylori infection compared to other studies. Abnormal nonulcerative and ulcerative changes constituted the dominant findings in $H$. pylori-positive patients. It seems that this lower prevalence of $H$. pylori may be due to an improvement in the socioeconomic status of the Iranian population.

\begin{tabular}{lc}
\hline Table 2. Pattern of Endoscopic Findings in H. pylori Infection \\
\hline Variables & Patients, No. (\%) \\
\hline Endoscopic findings & \\
\hline Group 1(normal) & $21(12.4)$ \\
Group 2 (abnormal nonulcerative) & $62(36.4)$ \\
Group 3 (ulcerative) & $29(17.1)$ \\
Group 4 (combination of groups 2 and 3) & $58(34.1)$ \\
\hline
\end{tabular}

\section{Acknowledgements}

We would gratefully like to thank all the patients who contributed to this study.

\section{Authors' Contributions}

Study concept and design: Laleh Mahmoudi, Mohammad Reza Fattahi and Ramin Niknam. Analysis and interpretation of data: Laleh Mahmoudi, Ramin Niknam, Mohammad Reza Fattahi, Mehrdad Seddigh and Amirreza Dehghanian. Drafting of the manuscript: Laleh Mahmoudi, Ramin Niknam and Mohammad Reza Fattahi. Critical revision of the manuscript for important intellectual content: Laleh Mahmoudi and Ramin Niknam. Statistical analysis: Mehrdad Seddigh. Study supervision: Laleh Mahmoudi and Ramin Niknam.

\section{Funding/Support}

The financial support of this research was provided by the Pharmaceutical Sciences Research Center (PSRC), Shiraz University of Medical Sciences, Shiraz, Iran.

\section{References}

1. Salih BA. Helicobacter pylori infection in developing countries: the burden for how long? Saudi J Gastroenterol. 2009;15(3):201-7.

2. Massarrat S, Saberi-Firoozi M, Soleimani A, Himmelmann GW Hitzges M, Keshavarz H. Peptic ulcer disease, irritable bowel syndrome and constipation in two populations in Iran. Eur J Gastroenterol Hepatol. 1995;7(5):427-33.

3. Malekzadeh R, Sotoudeh M, Derakhshan MH, Mikaeli J, Yazdanbod A, Merat S, et al. Prevalence of gastric precancerous lesions in Ardabil, a high incidence province for gastric adenocarcinoma in the northwest of Iran. JClin Pathol. 2004;57(1):37-42.

4. Go MF. Review article: natural history and epidemiology of Helicobacter pylori infection. Aliment Pharmacol Ther. 2002;16 Suppl 1:3-15.

5. Rowland M, Daly L, Vaughan M, Higgins A, Bourke B, Drumm B. Age-specific incidence of Helicobacter pylori. Gastroenterology. 2006;130(1):65-72.

6. Poddar U, Yachha SK. Helicobacter pylori in children: an Indian perspective. Indian Pediatr. 2007;44(10):761-70.

7. Mishra S, Singh V, Rao GR, Dixit VK, Gulati AK, Nath G. Prevalence of Helicobacter pylori in asymptomatic subjects--a nested PCR based study. Infect Genet Evol. 2008;8(6):815-9.

8. Brown LM. Helicobacter pylori: epidemiology and routes of transmission. Epidemiol Rev. 2000;22(2):283-97.

9. Goodman KJ, Correa P, Tengana Aux HJ, Ramirez H, DeLany JP, Guerrero Pepinosa O, et al. Helicobacter pylori infection in the Colombian Andes: a population-based study of transmission pathways. Am J Epidemiol.1996;144(3):290-9.

10. Chen J, Bu XL, Wang QY, Hu PJ, Chen MH. Decreasing serop- 
revalence of Helicobacter pylori infection during 1993-2003 in Guangzhou, southern China. Helicobacter. 2007;12(2):164-9.

11. Farshad S, Japoni A, Alborzi A, Zarenezhad M, Ranjbar R. Chang ing prevalence of Helicobacter pylori in south of Iran. Iran J Clin Infect Dis. 2010;5(2):65-9.

12. Alazmi WM, Siddique I, Alateeqi N, Al-Nakib B. Prevalence of Helicobacter pylori infection among new outpatients with dyspepsia in Kuwait. BMC Gastroenterol. 2010;10:14.

13. Yim JY, Kim N, Choi SH, Kim YS, Cho KR, Kim SS, et al. Seroprevalence of Helicobacter pylori in South Korea. Helicobacter. 2007;12(4):333-40.

14. Goh KL. Prevalence of and risk factors for Helicobacter pylori infection in a multi-racial dyspeptic Malaysian population undergoing endoscopy.J Gastroenterol Hepatol. 1997;12(6):S29-35.

15. Mapel D, Roberts M, Overhiser A, Mason A. The epidemiology, diagnosis, and cost of dyspepsia and Helicobacter pylori gastritis: a case-control analysis in the Southwestern United States. Helicobacter. 2013;18(1):54-65.
16. Miwa H, Ghoshal UC, Gonlachanvit S, Gwee KA, Ang TL, Chang FY, et al. Asian consensus report on functional dyspepsia.J Neurogastroenterol Motil. 2012;18(2):150-68.

17. Whitaker MJ, Brun J, Carelli F. Controversy and consensus in the management of upper gastrointestinal disease in primary care. The International Gastro Primary Care Group. Int J Clin Pract. 1997;51(4):239-43.

18. Knill-Jones RP. Geographical differences in the prevalence of dyspepsia. Scand J Gastroenterol Suppl.1991;182:17-24.

19. Amini E, Keshteli AH, Jazi MS, Jahangiri P, Adibi P. Dyspepsia in Iran: SEPAHAN Systematic Review No. 3. Int J Prev Med. 2012;3(Suppl1):S18-25.

20. Ahmed KS, Khan AA, Ahmed I, Tiwari SK, Habeeb A, Ahi JD, et al. Impact of household hygiene and water source on the prevalence and transmission of Helicobacter pylori: a South Indian perspective. Singapore Med J. 2007;48(6):543-9.

21. Soll AH. Pathogenesis of peptic ulcer and implications for therapy. N Engl J Med.1990;322(13):909-16. 